\title{
State estimation for discrete-time systems with generalized Lipschitz nonlinear dynamics
}

\author{
Dazhong Wang ${ }^{1}$, Fang Song ${ }^{2,3}$ and Wei Zhang ${ }^{1,2^{*}}$
}

\author{
"Correspondence: \\ wizzhang@gmail.com \\ ${ }^{1}$ College of Mechanical Engineering, \\ Shanghai University of Engineering \\ Science, Shanghai, 201620, China \\ ${ }^{2}$ Laboratory of Intelligent Control \\ and Robotics, Shanghai University \\ of Engineering Science, Shanghai, \\ 201620, China \\ Full list of author information is \\ available at the end of the article
}

\begin{abstract}
This paper considers the state estimation problem for a class of discrete-time systems with generalized Lipschitz nonlinear dynamics. Under the assumption that the system nonlinearities satisfy a quadratically inner-boundedness condition, we design both the full-order observer and the reduced-order observer for the discrete-time nonlinear system. Sufficient conditions ensuring the existence of full-order observers as well as reduced-order observers for such systems are established and formulated in terms of linear matrix inequality (LMI). Compared with some existing results, we remove the one-sided Lipschitz restrict and extend the classical Lipschitz observer design to a larger class of discrete-time nonlinear systems. A numerical example is included to illustrate the effectiveness of the proposed design.
\end{abstract}

Keywords: observer design; quadratically inner-boundedness; Lipschitz condition; linear matrix inequality (LMI); discrete-time nonlinear systems

\section{Introduction}

During the past two decades, the state estimation or observer design problem for nonlinear dynamic systems has received extensively research attention; see [1-14] and the references therein. This is partly due to the fact that knowledge of the state of a dynamic system plays a key role in many control problems. It is well known that state estimation can be used for control design, diagnosis or synchronization and unknown input recovery. However, designing a state observer for a general nonlinear system is not easy or even impossible. Many current research efforts are focused on some specialized classes of nonlinear systems. For instance, Arcak et al. [1,2] developed a circle-criterion approach to design observer for sector nonlinear systems. For Lipschitz nonlinear systems, the existence conditions of the full-order as well as the reduce-order observers were established in Rajamani [3] and Zhu and Han [4], respectively. Robust observers for Lipschitz nonlinear systems subject to disturbances were proposed in $[5,6]$. Nonlinear observer for neutral uncertain time-delay systems was addressed in [7]. Very recently, the classical Lipschitz nonlinear observer design has been extended to the one-sided Lipschitz case; see e.g. [8-14].

It should be noted that most of the above-mentioned works are concerned on continuous-time nonlinear systems. Generally, the state estimation problem for discrete-time nonlinear systems has received little attention. Moreover, in the existing literature there

(c) 2015 Wang et al. This article is distributed under the terms of the Creative Commons Attribution 4.0 International License (http://creativecommons.org/licenses/by/4.0/), which permits unrestricted use, distribution, and reproduction in any medium, provided you give appropriate credit to the original author(s) and the source, provide a link to the Creative Commons license, and indicate if changes were made. 
have been several useful observer design approaches for some specialized classes of discrete-time nonlinear systems [15-23]. For example, Ibrir [15] proposed the circlecriterion approach to discrete-time nonlinear observer design. In [16] and [17], the authors considered the observer design for discrete-time Lipschitz nonlinear systems. Motivated by the Arcak-type observer design [1, 2], Zemouche and Boutayeb [18] provided a unified observer design method for discrete-time Lipschitz systems and extended it to $H_{\infty}$ synchronization and unknown input recovery. An LMI approach was proposed by Wang et al. [19] to design state observer for discrete-time Lipschitz descriptor systems. In [20] the authors considered an observer design for discrete-time epidemic models. A new reduced-order observer normal form for nonlinear discrete-time systems was provided in [21].

Very recently, several authors have considered the observer design for one-sided Lipschitz nonlinear systems in the discrete-time case. Both full-order and reduced-order observer designs were studied in Benallouch et al. [22]. In fact, they have developed an LMI-based design approach to deal with the state estimation problem of one-sided Lipschitz discrete-time systems. Zhang et al. [23] investigated the same problem and proposed a simple observer synthesis condition to ensure the asymptotic convergence. It should be emphasized that the systems considered by Benallouch et al. [22] and Zhang et al. [23] are actually a subset of one-sided Lipschitz nonlinear systems (see Figure 1 below). More precisely, the systems are assumed to simultaneously satisfy the one-sided Lipschitz condition and the quadratically inner-bounded condition. This assumption may lead to more conservative results and bring additional restrictions on the system model. How to reduce the conservatism in the existing results of observer design of nonlinear systems is still an open problem. This motivates our present research.

In this paper, we focus on state observer design for a general class of nonlinear discretetime systems that satisfies the quadratically inner-bounded condition only. The main contributions of this paper are two folds. First, we remove the one-sided Lipschitz restriction and only need the assumption of quadratically inner-bounded condition. Note that the quadratically inner-bounded condition includes the classical Lipschitz condition as a special case; see e.g. Figure 1 below. Therefore, we extend the state observer design to a larger class of discrete-time nonlinear systems. Second, some simple stability conditions are obtained for both full-order and reduced-order observer designs. In our approach, the observer designs are formulated as an LMI feasible problem, which is easily solved by standard convex optimization algorithms. An example on the single-link flexible joint robot is given to illustrate the effectiveness of the proposed design.

Notations: $\mathbb{R}^{n}$ denotes the $n$-dimensional real Euclidean space. $\langle\cdot, \cdot\rangle$ represents the inner product in $\mathbb{R}^{n}$, i.e., for given $x, y \in \mathbb{R}^{n}$, then $\langle x, y\rangle=x^{T} y$, where $x^{T}$ is the transpose of the column vector $x \in \mathbb{R}^{n}$. $\|\cdot\|$ denotes the Euclidean norm on $\mathbb{R}^{n}$. For a symmetric matrix $P$, $P>0(P<0)$ means that the matrix is positive definite (negative definite). In symmetric block matrices, we use an asterisk $*$ to represent a term induced by symmetry. I represents an identity matrix with appropriate dimension.

\section{Problem statement and preliminaries}

In this paper, we consider the class of discrete-time nonlinear systems described by

$$
\left\{\begin{array}{l}
x(k+1)=A x(k)+B u(k)+f(x(k), y(k)), \\
y(k)=C x(k),
\end{array}\right.
$$


Figure 1 The Lipschitz, one-sided Lipschitz, and quadratically inner-bounded function sets [24].

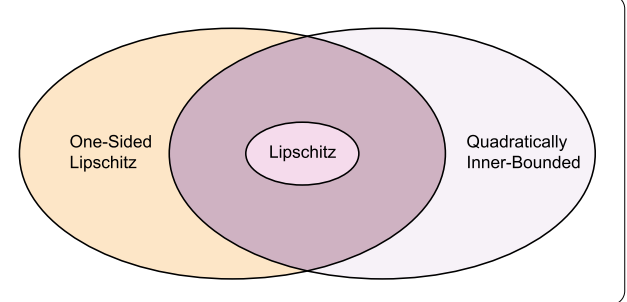

where $x(k) \in \mathbb{R}^{n}$ is the state vector and $y(k) \in \mathbb{R}^{p}$ is the linear measured output. $A, B$ and $C$ are constant matrices of appropriate dimensions. $f: \mathbb{R}^{n} \times \mathbb{R}^{p} \rightarrow \mathbb{R}^{n}$ is a real nonlinear vector field, which is assumed to satisfy the following quadratically inner-bonded condition [22].

Assumption 1 (see e.g. $[9,22]) f$ is quadratically inner-bounded with respect to $x(k)$, i.e., for all $x, \hat{x} \in \mathbb{R}^{n}$, there exist $\beta, \gamma \in \mathbb{R}$ such that

$$
\|f(x, y(k))-f(\hat{x}, y(k))\|^{2} \leq \beta\|x-\hat{x}\|^{2}+\gamma\langle x-\hat{x}, f(x, y(k))-f(\hat{x}, y(k))\rangle .
$$

It is clear that any Lipschitz function is also quadratically inner-bounded corresponding to $\beta>0$ and $\gamma=0$. Consequently, Lipschitz continuity implies quadratic innerboundedness, but the converse is not true $[8,9]$. It should be emphasized that $\beta$ and $\gamma$ in (2) can be any real number and are not necessarily positive. Therefore, the system considered in the paper includes the well-known Lipschitz nonlinear system as a special case (see Figure 1).

For the purpose of comparison, we introduce the following two assumptions, which are commonly used in the recent literature for observer design of nonlinear systems. For further details, we refer the interested reader to $[8,9,22]$.

Assumption 2 (see e.g. [9]) $f$ is Lipschitz with respect to $x(k)$, i.e., for all $x, \hat{x} \in \mathbb{R}^{n}$, there exists a scalar $\lambda>0$ such that

$$
\|f(x, y(k))-f(\hat{x}, y(k))\| \leq \lambda\|x-\hat{x}\| .
$$

Assumption 3 (see e.g. $[9,22]) f$ is one-sided Lipschitz with respect to $x(k)$, i.e., for all $x, \hat{x} \in \mathbb{R}^{n}$, there exists a scalar $\rho \in \mathbb{R}$ such that

$$
\langle x-\hat{x}, f(x, y(k))-f(\hat{x}, y(k))\rangle \leq \rho\|x-\hat{x}\|^{2}
$$

Notice that Assumption 2 is the well-known Lipschitz condition, while Assumption 3 is the so-called one-sided Lipschitz condition. It is worth mentioning that the one-sided Lipschitz condition has been frequently employed in the study of synchronization of complex networks [25, 26]. Moreover, as shown in [8] and [9], the one-sided Lipschitz condition implies the Lipschitz condition but the converse is not true. Figure 1 shows the relation between the Lipschitz, one-sided Lipschitz, and quadratically inner-bounded function sets [24].

We end this section by introducing a useful lemma. 
Lemma 1 (The Schur complement lemma; see e.g. [27]) For a real symmetric matrix $\Sigma$, the following assertions are equivalent:

(1) $\Sigma:=\left[\begin{array}{cc}\Sigma_{11} & \Sigma_{12} \\ \Sigma_{12}^{T} & \Sigma_{22}\end{array}\right]<0$.

(2) $\Sigma_{11}<0$, and $\Sigma_{22}-\Sigma_{12}^{T} \Sigma_{11}^{-1} \Sigma_{12}<0$.

(3) $\Sigma_{22}<0$, and $\Sigma_{11}-\Sigma_{12} \Sigma_{22}^{-1} \Sigma_{12}^{T}<0$.

\section{Full-order observer design}

In this section, we consider the full-order observer design for system (1) under Assumption 1. As usual, we consider a Luenberger-like observer for system (1) in the form of

$$
\left\{\begin{array}{l}
\hat{x}(k+1)=A \hat{x}(k)+B u(k)+f(\hat{x}(k), u(k))+L(y(k)-\hat{y}(k)), \\
\hat{y}(k)=C \hat{x}(k),
\end{array}\right.
$$

where $\hat{x}(k)$ denotes the estimate of the state $x(k)$. Our design goal is to find a gain matrix $L$ such that the estimation error $e(k):=x(k)-\hat{x}(k)$ converges asymptotically toward zero. From (1) and (5), the dynamics of the estimation error is governed by

$$
e(k+1)=(A-L C) e(k)+\Delta f_{k}
$$

where $\Delta f_{k}:=f(x(k), y(k))-f(\hat{x}(k), y(k))$.

Now, we have the following conclusion.

Theorem 1 Suppose that system (1) satisfies Assumption 1 and the observer has the form of (5). Then the error dynamics is asymptotically stable if there exist matrices $P>0$ and $R$ with appropriate dimensions and a scalar $\omega>0$ such that the following LMI is feasible:

$$
\left[\begin{array}{ccc}
-P+2 \omega \beta I & A^{T} P-C^{T} R+\omega \gamma I & A^{T} P-C^{T} R \\
* & P-2 \omega I & 0 \\
* & * & -P
\end{array}\right]<0 .
$$

The resulting observer gain matrix $L$ is given by $L=P^{-1} R^{T}$.

Proof For the estimation error dynamics (6), let us consider the Lyapunov function candidate $V(k)=e^{T}(k) P e(k)$. Then the difference of $V(k)$ along the trajectories of (6) is given by

$$
\Delta V_{k}:=V(k+1)-V(k)=e^{T}(k+1) P e(k+1)-e^{T}(k) P e(k) .
$$

By Assumption 1,

$$
\Delta f_{k}^{T} \Delta f_{k} \leq \beta e^{T}(k) e(k)+\gamma e^{T}(k) \Delta f_{k}
$$

It then follows from (9) that

$$
2 \omega \beta e^{T}(k) e(k)+2 \omega \gamma e^{T}(k) \Delta f_{k}-2 \omega \Delta f_{k}^{T} \Delta f_{k} \geq 0
$$


where $\omega>0$ is a scalar. Adding the left-hand side of (10) to $\Delta V_{k}$ yields

$$
\begin{aligned}
\Delta V_{k} \leq & e^{T}(k+1) \operatorname{Pe}(k+1)-e^{T}(k) \operatorname{Pe}(k)+2 \omega \beta e^{T}(k) e(k) \\
& +2 \omega \gamma e^{T}(k) \Delta f_{k}-2 \omega \Delta f_{k}^{T} \Delta f_{k} \\
= & \xi_{k}^{T} \Omega \xi_{k},
\end{aligned}
$$

where $\xi_{k}^{T}=\left[e(k) \Delta f_{k}\right]^{T}$ and

$$
\Omega=\left[\begin{array}{cc}
(A-L C)^{T} P(A-L C)-P+2 \omega \beta I & (A-L C)^{T} P+\omega \gamma I \\
P(A-L C)+\omega \gamma I & P-2 \omega I
\end{array}\right] .
$$

Applying Lemma $1, \Omega<0$ is equivalent to

$$
\Gamma=\left[\begin{array}{ccc}
-P+2 \omega \beta I & (A-L C)^{T} P+\omega \gamma I & (A-L C)^{T} P \\
* & P-2 \omega I & 0 \\
* & * & -P
\end{array}\right]<0 .
$$

By denoting $R=L^{T} P$, the condition (7) implies $\Gamma<0$. Therefore, we have $\Delta V_{k}<0$ for all $e(k) \neq 0$ if $(7)$ is satisfied. This completes the proof.

Since the quadratically inner-bounded condition include the Lipschitz condition as a special case, we immediately have Corollary 1.

Corollary 1 Suppose that system (1) satisfies Assumption 2 and the observer has the form of (5). Then the error dynamics is asymptotically stable if there exist matrices $P>0$ and $R$ with appropriate dimensions and a scalar $\omega>0$ such that the following LMI is feasible:

$$
\left[\begin{array}{ccc}
-P+\omega \lambda^{2} I & A^{T} P-C^{T} R & A^{T} P-C^{T} R \\
* & P-\omega I & 0 \\
* & * & -P
\end{array}\right]<0 .
$$

The resulting observer gain matrix $L$ is given by $L=P^{-1} R^{T}$.

\section{Reduced-order observer design}

In this section, we address the reduced-order observer design problem for system (1) under Assumption 1. Note that our design is inspired by the approach developed in [17] and [22], but we remove the one-sided Lipschitz restriction and provide a simple observer synthesis condition. Let $\xi(k)$ denote the reduced state vector to be estimated. Without loss of generality, assume

$$
z(k)=H x(k)
$$

where $H \in \mathbb{R}^{(n-p) \times n}$ is a matrix so that $\left[\begin{array}{c}H \\ C\end{array}\right]$ is nonsingular with

$$
\left[\begin{array}{l}
H \\
C
\end{array}\right]^{-1}=\left[\begin{array}{ll}
N & M
\end{array}\right]
$$


We then have

$$
x(k)=N z(k)+M y(k) .
$$

From (1), (14), and (16), we obtain the following nonlinear reduced form:

$$
\begin{aligned}
z(k+1) & =H x(k+1) \\
& =H(A x(k)+B u(k)+f(x(k), y(k))) \\
& =A_{Z} z(k)+H B u(k)+H g(z(k), y(k))+B_{Z} y(k),
\end{aligned}
$$

where $A_{Z}:=H A N, B_{Z}:=H A M$, and $g(z(k), y(k)):=f(N z(k)+M y(k), y(k))$.

Inspired by [22], we design a reduced-order observer corresponding to (17) as follows:

$$
\left\{\begin{array}{l}
\hat{z}(k+1)=A_{Z} \hat{z}(k)+H B u(k)+B_{Z} y(k)+H g(\hat{z}(k), y(k))+K(y(k+1)-C \zeta(k)), \\
\zeta(k)=A N \hat{z}(k)+A M y(k)+g(\hat{z}(k), y(k)), \\
\hat{x}(k)=N \hat{z}(k)+M y(k) .
\end{array}\right.
$$

Denoting the estimator error by $\varepsilon(k):=z(k)-\hat{z}(k)$ and letting $C_{Z}:=C A N$, we have

$$
\begin{aligned}
K(y(k+1)-C \zeta(k)) & =K C(x(k+1)-\zeta(k)) \\
& =K C(A x(k)+f(x(k), y(k))-\zeta(k)) \\
& =K C(A N z(k)+A M y(k)+f(N z(k)+M y(k), y(k))-\zeta(k)) \\
& =K C_{Z} \varepsilon(k)+K C \Delta g_{k},
\end{aligned}
$$

where $\Delta g_{k}:=g(z(k), y(k))-g(\hat{z}(k), y(k))$.

From (17)-(19), we know that the dynamics of the estimation error is governed by

$$
\varepsilon(k+1)=\left(A_{Z}-K C_{Z}\right) \varepsilon(k)+(H-K C) \Delta g_{k} .
$$

Now, we have the following theorem.

Theorem 2 Under Assumption 1, the proposed reduced-order observer (18) is an asymptotic observer for system (1) if there exist matrices $P>0$ and $K$ of appropriate dimensions and a scalar $\omega>0$ such that the following matrix inequality is feasible:

$$
\left[\begin{array}{ccc}
-N^{T} P N+2 \omega \beta N^{T} N & \omega \gamma N^{T} & \left(A_{Z}-K C_{Z}\right)^{T} N^{T} P \\
* & -2 \omega I & (H-K C)^{T} N^{T} P \\
* & * & -P
\end{array}\right]<0 .
$$

Proof Notice that $e(k)=N \varepsilon(k)$. For the error dynamics (20), we also consider the Lyapunov function candidate $V(k)=e^{T}(k) P e(k)$, i.e., $V(k)=\varepsilon^{T}(k) N^{T} P N \varepsilon(k)$. Then the difference of $V(k)$ along the trajectories of (20) is given by

$$
\Delta V_{k}:=V(k+1)-V(k)=\varepsilon^{T}(k+1) N^{T} P N \varepsilon(k+1)-\varepsilon^{T}(k) N^{T} P N \varepsilon(k) .
$$


By Assumption 1, the nonlinear function $f(x(k), y(k))$ is quadratically inner-bounded, then also the function $g(z(k), y(k))$ is quadratically inner-bounded with constants $\beta_{g}$ and $\gamma_{g}$. In fact, from Assumption 1, we can deduce

$$
2 \omega \beta e^{T}(k) e(k)+2 \omega \gamma e^{T}(k) \Delta f_{k}-2 \omega \Delta f_{k}^{T} \Delta f_{k} \geq 0,
$$

where $\omega>0$ is a scalar. Note that $e(k)=N \varepsilon(k)$ and $\Delta f_{k}=\Delta g_{k}$. It follows from (23) that

$$
2 \omega \beta \varepsilon^{T}(k) N^{T} N \varepsilon(k)+2 \omega \gamma \varepsilon^{T}(k) N^{T} \Delta g_{k}-2 \omega \Delta g_{k}^{T} \Delta g_{k} \geq 0 .
$$

Adding the left-hand side of (24) to $\Delta V_{k}$ yields

$$
\begin{aligned}
\Delta V_{k} \leq & \varepsilon^{T}(k+1) N^{T} P N \varepsilon(k+1)-\varepsilon^{T}(k) N^{T} P N \varepsilon(k)+2 \omega \beta \varepsilon^{T}(k) N^{T} N \varepsilon(k) \\
& +2 \omega \gamma \varepsilon^{T}(k) N^{T} \Delta g_{k}-2 \omega \Delta g_{k}^{T} \Delta g_{k} \\
= & \chi_{k}^{T} \Pi \chi_{k},
\end{aligned}
$$

where $\chi_{k}^{T}=\left[\varepsilon(k) \Delta g_{k}\right]^{T}$, and

$$
\begin{aligned}
& \Pi=\left[\begin{array}{c}
\left(A_{Z}-K C_{Z}\right)^{T} \\
(H-K C)^{T}
\end{array}\right] N^{T} P N\left[A_{Z}-K C_{Z} \quad H-K C\right] \\
& +\left[\begin{array}{cc}
-N^{T} P N+2 \omega \beta N^{T} N & \omega \gamma N^{T} \\
& -2 \omega I
\end{array}\right] .
\end{aligned}
$$

Note that $\Delta V_{k}<0$ if $\Pi<0$. Using Lemma $1, \Pi<0$ is equivalent to

$$
\left[\begin{array}{ccc}
-N^{T} P N+2 \omega \beta N^{T} N & \omega \gamma N^{T} & \left(A_{Z}-K C_{Z}\right)^{T} N^{T} P \\
* & -2 \omega I & (H-K C)^{T} N^{T} P \\
* & * & -P
\end{array}\right]<0 .
$$

Therefore, if the matrix inequality (21) has a feasible solution, we have $\Delta V_{k}<0$ for all $\varepsilon(k) \neq 0$. By the standard Lyapunov theorem, we know that the estimation error system is asymptotically stable, which means (18) is an asymptotic reduced-order observer for system (1). This completes the proof.

Remark 1 Compared with the full-order or the reduced-order observer design in [22], the paper removes the one-sided Lipschitz restriction, which significantly reduces the conservatism and complexity of the designs. In fact, in Theorems 1 and 2 , we only assume that $f$ satisfies the quadratically inner-bounded condition (2) and do not employ the one-sided Lipschitz condition (3).

Remark 2 It should be noted that (21) is not an LMI. To make it more tractable, we can formulate it into an LMI by letting $P=\alpha I$ for a prior given scalar $\alpha>0$. In this case, (21) becomes

$$
\left[\begin{array}{ccc}
-\alpha N^{T} N+2 \omega \beta N^{T} N & \omega \gamma N^{T} & \alpha\left(A_{Z}-K C_{Z}\right)^{T} N^{T} \\
* & -2 \omega I & \alpha(H-K C)^{T} N^{T} \\
* & * & -\alpha I
\end{array}\right]<0 .
$$


Similarly, we have Corollary 2 , since the quadratically inner-bounded condition includes the Lipschitz condition as a special case.

Corollary 2 Under Assumption 2, the proposed reduced-order observer (18) is an asymptotic observer for system (1) if there exist matrices $P>0$ and $K$ of appropriate dimensions and a scalar $\omega>0$ such that the following matrix inequality is feasible:

$$
\left[\begin{array}{ccc}
-N^{T} P N+2 \omega \lambda^{2} N^{T} N & 0 & \left(A_{Z}-K C_{Z}\right)^{T} N^{T} P \\
* & -2 \omega I & (H-K C)^{T} N^{T} P \\
* & * & -P
\end{array}\right]<0 .
$$

\section{Illustrative example}

In this section gives a numerical example to illustrate the applications of the proposed observer design. For convenience, we take the well-known single-link flexible joint robotic system as an example [3, 4]. The continuous-time model of the system is described by

$$
\left\{\begin{array}{l}
\dot{x}(t)=A_{c} x(t)+B_{c} u(t)+f_{c}(x(t), y(t)) \\
y(t)=C_{c} x(t)
\end{array}\right.
$$

where

$$
\begin{aligned}
A_{c} & =\left[\begin{array}{cccc}
0 & 1 & 0 & 0 \\
-48.6 & -1.26 & 48.6 & 0 \\
0 & 0 & 0 & 10 \\
1.95 & 0 & -1.95 & 0
\end{array}\right], \\
B_{c} & =\left[\begin{array}{c}
0 \\
21.6 \\
0 \\
0
\end{array}\right],
\end{aligned}
$$

and

$$
f_{c}(x, y)=\left[\begin{array}{c}
0 \\
0 \\
0 \\
-0.333 \sin \left(x_{1}\right)
\end{array}\right] .
$$

Let $T_{e}$ be the sample time. Then by using the Euler discretized approach on system (28), we can derive the following discrete-time system model:

$$
\left\{\begin{array}{l}
x(k+1)=A x(k)+B u(k)+f(x(k), y(k)), \\
y(k)=C x(k)
\end{array}\right.
$$

where

$$
A=I_{4}+T_{e} A_{c}, \quad B=T_{e} B_{c}, \quad C=C_{c}, \quad f(x(k), y(k))=T_{e} f_{c}(x(t), y(t)) .
$$


It is easy to verify that $f(x(k), y(k))$ is quadratically inner-bounded with $\beta=\left(0.333 T_{e}\right)^{2}$ and $\gamma=0$. Let the sample time $T_{e}=0.1[s]$. To design the full-order observer, we need to solve the LMI (7). By using the Matlab LMI tool, we get

$$
\begin{aligned}
P & =\left[\begin{array}{cccc}
16.3522 & 0 & 0 & 0 \\
0 & 1.4394 & -4.0400 & 1.9195 \\
0 & -4.0400 & 14.8752 & -10.0169 \\
0 & 1.9195 & -10.0169 & 15.9081
\end{array}\right], \\
R & =\left[\begin{array}{cccc}
16.3522 & -6.6211 & 17.6813 & -6.2265 \\
1.6352 & 1.9279 & -4.0321 & 0.9475
\end{array}\right], \quad \omega=16.7916 .
\end{aligned}
$$

Therefore, the full-order observer gain matrix $L$ is given by

$$
L=P^{-1} R^{T}=\left[\begin{array}{cc}
1.0000 & 0.1000 \\
-4.8600 & 2.4927 \\
0 & 0.4228 \\
0.1950 & 0.0250
\end{array}\right]
$$

On the other hand, the reduced-order observer can be designed by using Theorem 2 . With

$$
H=\left[\begin{array}{llll}
0 & 0 & 1 & 0 \\
0 & 0 & 0 & 1
\end{array}\right],
$$

we have

$$
N=\left[\begin{array}{ll}
0 & 0 \\
0 & 0 \\
1 & 0 \\
0 & 1
\end{array}\right], \quad M=\left[\begin{array}{ll}
1 & 0 \\
0 & 1 \\
0 & 0 \\
0 & 0
\end{array}\right] .
$$

Let $\alpha=0.001$. By solving the LMI (21), we obtain the reduced-order observer gain matrix

$$
K=\left[\begin{array}{cc}
0 & 0.2057 \\
0 & -0.0401
\end{array}\right] .
$$

\section{Conclusion}

We have addressed the state estimation problem for a general class of nonlinear discretetime systems that satisfies the quadratically inner-bounded condition. The system under consideration need not satisfy the one-sided Lipschitz restriction, which is a common assumption in some recent literature on observer design for nonlinear discrete-time systems. We considered both the full-order and the reduced-order observer designs and formulated the observer synthesis condition as an LMI formulation. Finally, we used an example on the single-link flexible joint robotic system to illustrate the effectiveness of the proposed design. 
Authors' contributions

All authors contributed equally to the writing of this paper. All authors read and approved the final manuscript.

\section{Author details}

${ }^{1}$ College of Mechanical Engineering, Shanghai University of Engineering Science, Shanghai, 201620, China. ${ }^{2}$ Laboratory of Intelligent Control and Robotics, Shanghai University of Engineering Science, Shanghai, 201620, China. ${ }^{3}$ State Key Laboratory of Robotics and System, Harbin Institute of Technology, Harbin, 150001, China.

\section{Acknowledgements}

This work was supported in part by the National Natural Science Foundation of China under Grant 51505273, the State Key Laboratory of Robotics and System (HIT) under Grant SKLRS-2014-MS-10, the Jiangsu Provincial Key Laboratory of Advanced Robotics Fund Projects under Grant JAR201401, and the Foundation of Shanghai University of Engineering Science under Grant nhky-2015-06.

Received: 4 August 2015 Accepted: 24 September 2015 Published online: 06 October 2015

\section{References}

1. Arcak, M, Kokotovic, P: Nonlinear observers: a circle criterion design and robustness analysis. Automatica 37, 1923-1930 (2001)

2. Fan, X, Arack, M: Observer design for systems with multivariable monotone nonlinearities. Syst. Control Lett. 50, 319-330 (2003)

3. Rajamani, R: Observers for Lipschitz nonlinear systems. IEEE Trans. Autom. Control 43(3), 397-401 (1998)

4. Zhu, F, Han, Z: A note on observers for Lipschitz nonlinear systems. IEEE Trans. Autom. Control 47(10), 1751-1754 (2002)

5. Chen, $M$, Chen, C: Robust nonlinear observer for Lipschitz nonlinear systems subject to disturbances. IEEE Trans. Autom. Control 52(12), 2365-2369 (2007)

6. Zhang, W, Xie, H, Su, H, Zhu, F: Improved results on generalized robust $H_{\infty}$ filtering for Lipschitz descriptor nonlinear systems with uncertainties. IET Control Theory Appl. 9(14), 2107-2114 (2015)

7. Dong, Y, Li, T, Zhang, X: Stability analysis of nonlinear observer for neutral uncertain time-delay systems. Adv. Differ. Equ. 2014, 133 (2014)

8. Hu, G: Observers for one-sided Lipschitz non-linear systems. IMA J. Math. Control Inf. 23(4), 395-401 (2006)

9. Abbaszadeh, M, Marquez, HJ: Nonlinear observer design for one-sided Lipschitz systems. In: Proceedings of the American Control Conference, pp. 5284-5289 (2010)

10. Xu, M, Hu, G, Zhao, Y: Reduced-order observer for one-sided Lipschitz nonlinear systems. IMA J. Math. Control Inf. 26, 299-317 (2009)

11. Zhang, W, Su, H, Liang, Y, Han, Z: Nonlinear observer design for one-sided Lipschitz nonlinear systems: an linear matrix inequality approach. IET Control Theory Appl. 6(9), 1297-1303 (2012)

12. Zhang, W, Su, H, Wang, H, Han, Z: Full-order and reduced-order observers for one-sided Lipschitz nonlinear systems using Riccati equations. Commun. Nonlinear Sci. Numer. Simul. 17(12), 4968-4977 (2012)

13. Zhao, Y, Tao, J, Shi, N: A note on observer design for one-sided Lipschitz nonlinear systems. Syst. Control Lett. 59, 66-71 (2010)

14. Zhang, W, Su, H, Zhu, F, Azar, G: Unknown input observer design for one-sided Lipschitz nonlinear systems. Nonlinear Dyn. 79, 1469-1479 (2015)

15. Ibrir, S: Circle-criterion approach to discrete-time nonlinear observer design. Automatica 43, 1432-1441 (2007)

16. Abbaszadeh, $\mathrm{M}$, Marquez, $\mathrm{HJ}$ : Robust $H_{\infty}$ observer design for sampled-data Lipschitz nonlinear systems with exact and Euler approximate models. Automatica 44, 799-806 (2008)

17. Zemouche, A, Boutayeb, M: Observer design for Lipschitz nonlinear systems: the discrete-time case. IEEE Trans. Circuits Syst. II, Express Briefs 53(8), 777-781 (2006)

18. Zemouche, A, Boutayeb, M: Nonlinear-observer-based $H_{\infty}$ synchronization and unknown input recovery. IEEE Trans. Circuits Syst. I, Regul. Pap. 56(8), 1720-1731 (2009)

19. Wang, Z, Shen, Y, Zhang, X, Wang, Q: Observer design for discrete-time descriptor systems: an LMI approach. Syst. Control Lett. 61, 683-687 (2012)

20. Ibeas, A, Sen, M, Alonso-Quesada, S, Zamani, I: Stability analysis and observer design for discrete-time SEIR epidemic models. Adv. Differ. Equ. 2015, 122 (2015)

21. Boutat, D, Boutat-Baddas, L, Darouach, M: A new reduced-order observer normal form for nonlinear discrete time systems. Syst. Control Lett. 61, 1003-1008 (2012)

22. Benallouch, M, Boutayeb, M, Zasadzinski, M: Observer design for one-sided Lipschitz discrete-time systems. Syst. Control Lett. 61, 879-886 (2012)

23. Zhang, W, Su, H, Zhu, F, Yue, D: A note on observers for discrete-time Lipschitz nonlinear systems. IEEE Trans. Circuits Syst. II, Express Briefs 59(2), 123-127 (2012)

24. Abbaszadeh, M, Marquez, HJ: Design of nonlinear state observers for one-sided Lipschitz systems (2013). arXiv:1302.5867

25. Yu, W, DeLellis, P, Chen, G, Bernardo, M, Kurths, J: Distributed adaptive control of synchronization in complex networks. IEEE Trans. Autom. Control 57, 2153-2158 (2012)

26. Su, H, Chen, G, Wang, X, Lin, Z: Adaptive second-order consensus of networked mobile agents with nonlinear dynamics. Automatica 47, 368-375 (2011)

27. Boyd, S, Ghaoui, LE, Feron, E, Balakrishnan, V: Linear Matrix Inequalities in System and Control Theory. SIAM, Philadelphia (1994) 